\title{
FÓSFORO REMANESCENTE EM ARGILA E SILTE RETIRADOS DE LATOSSOLOS APÓS PRÉ-TRATAMENTOS NA ANÁLISE TEXTURAL (1)
}

\author{
Guilherme Kangussú Donagemma ${ }^{(2)}$, Hugo Alberto Ruiz ${ }^{(3)}$, \\ Víctor Hugo Alvarez V. ${ }^{(3)}$, João Carlos $\mathrm{Ker}^{(3)}$ \& Maurício Paulo \\ Ferreira Fontes $^{(3)}$
}

\section{RESUMO}

\begin{abstract}
Alguns Latossolos podem apresentar problemas de dispersão, devido a grupamentos de microagregados de alta estabilidade. Esses grupamentos, muito argilosos, são parcialmente desagregados pela dispersão química e mecânica, contribuindo, pelo seu tamanho, para superestimar a proporção de silte do solo analisado. Para reduzir a proporção de pseudo-componentes aplicam-se prétratamentos, cuja eficiência pode ser testada pela capacidade de retenção de fosfatos. Assim, realizou-se um ensaio em laboratório com objetivo de verificar o uso da determinação do fósforo remanescente (Prem) como medida da eficiência de pré-tratamentos na análise textural de Latossolos. Os tratamentos corresponderam a um arranjo fatorial $7 \times 2^{2}$, num delineamento em blocos casualizados, com quatro repetições. Os fatores em estudo foram as amostras de sete Latossolos dispersos com $\mathrm{NaOH} 0,1 \mathrm{~mol} \mathrm{~L}^{-1}$, sem e com $\left(2^{2}\right)$ pré-tratamentos para a remoção da matéria orgânica (-MO) e dos óxidos de Fe e Al mal cristalizados (-Ox). Determinou-se o Prem em amostras da fração argila e silte dispersas uma vez e da fração silte dispersa uma segunda vez. A determinação do Prem é eficiente em mostrar diferenças entre os tratamentos aplicados, evidenciando alterações na capacidade de retenção de fosfatos, que foi incrementada no tratamento-MO, por maior exposição de componentes inorgânicos, e reduzida no tratamento-Ox, por diminuir a proporção de óxidos mal cristalizados.
\end{abstract}

Termos de indexação: análise granulométrica, matéria orgânica, óxidos de Fe.

\footnotetext{
(1) Parte da Dissertação de Mestrado do primeiro autor apresentada à Universidade Federal de Viçosa - UFV. Recebido para publicação em agosto de 2007 e aprovado em abril de 2008.

(2) Pesquisador da Empresa Brasileira de Pesquisa Agropecuária - EMBRAPA Solos. Rua Jardim Botânico 1.024, Jardim Botânico, CEP 22460-000 Rio de Janeiro (RJ). E-mail: donagemma@cnps.embrapa.br

(3) Professor do Departamento de Solos, Universidade Federal de Viçosa - UFV. CEP 36570-000 Viçosa (MG). Bolsista do CNPq. E-mails: hruiz@ufv.br; vhav@ufv.br; jcker@ufv.br; mpfontes@ufv.br
} 


\title{
SUMMARY: SOLUTION EQUILIBRIUM PHOSPHORUS IN CLAY AND SILT FRACTIONS OF OXISOLS AFTER PARTICLE SIZE ANALYSIS PRE-TREATMENTS
}

\begin{abstract}
The presence of highly stable microaggregates can hamper soil dispersion in some Oxisols. These very clayey groupings may be not completely disaggregated by chemical and mechanical dispersion. Due to their size, the silt proportion of the soil is overestimated. A laboratory experiment was conducted to verify the use of the solution equilibrium $P$ (SEP) to evaluate the response to particle size analysis pre-treatments in Oxisols. The treatments were arranged in a $7 \times 2^{2}$ factorial design, in randomized blocks with four replications. The factors were samples of seven Oxisols dispersed with $0.1 \mathrm{~mol} \mathrm{~L}^{-1} \mathrm{NaOH}$, with and without pre-treatment to remove organic matter (-OM) and poorly crystallized iron oxides (-Ox). SEP was determined in samples of the dispersed clay and silt fractions and in the silt fraction, after a second dispersion with $0.1 \mathrm{~mol} \mathrm{~L} \mathrm{~L}^{-1} \mathrm{NaOH}$. It was observed that $S E P$ is sensitive enough to identify differences between the treatments applied. This indicates the usefulness of SEP as an auxiliary determination in soil dispersion studies.
\end{abstract}

Index terms: soil texture, organic matter, iron oxides.

\section{INTRODUÇÃO}

O fósforo remanescente (Prem) mede a quantidade de $\mathrm{P}$ que permanece em solução de equilíbrio em resposta a uma concentração de $\mathrm{P}$ adicionada ao solo. Essa determinação proposta por Bache \& Williams (1971) tem sido uma solução prática para se obter um índice do fator capacidade de P dos solos (Novais \& Smyth, 1999). No Brasil, a determinação do Prem é feita utilizando uma solução de $\mathrm{CaCl}_{2} 10 \mathrm{mmol} \mathrm{L}^{-1}$ contendo de 30 a $60 \mathrm{mg} \mathrm{L}^{-1}$ de $\mathrm{P}$, numa relação solo/ solução de 1:10. Atualmente, a concentração de $60 \mathrm{mg} \mathrm{L}^{-1}$ de $\mathrm{P}$ tem sido considerada padrão (Novais $\&$ Smyth, 1999).

O Prem também tem sido utilizado para definir as concentrações de P nas soluções a serem colocadas em equilíbrio para determinar a capacidade máxima de adsorção de fosfatos (Alvarez V. \& Fonseca, 1990), pois apresenta estreita correlação com esta característica e também com a capacidade tampão de fosfatos (Novais, 1977; Alvarez V., 1982; Bahia Filho, 1982; Resende, 1983; Muniz, 1983; Muniz et al., 1987; Ker, 1995; Fernández R., 1995). A quantidade de P que permanece na solução depende da atuação combinada da concentração de $\mathrm{P}$ adicionada e do tempo de contato (Alvarez V. et al., 2001) e da capacidade de adsorção de fosfatos do solo, que, por sua vez, é dependente da quantidade de MO, da textura e da mineralogia da fração argila (Novais \& Smyth, 1999). Quanto maior a quantidade de MO, em geral menor é a adsorção de fosfatos e maior é o Prem, uma vez que ácidos orgânicos de baixo peso molecular, como malato, oxalato e citrato, podem bloquear sítios de adsorção do fosfato (Lopez-Hernandez et al., 1986; Violante et al., 1991; Silva et al., 1997; Bhatti et al., 1998). Com a remoção de parte da MO, há exposição de cargas positivas que adsorvem o fosfato, diminuindo assim sua concentração na solução de equilíbrio.
Considerando-se, apenas, a influência da textura, espera-se, em razão da diferença de superfície específica entre as frações texturais, a seguinte ordem de adsorção de fosfatos: argila $>$ silte $>$ areia fina $>$ areia grossa. Em relação à mineralogia da fração argila, os óxidos de $\mathrm{Fe}$ e Al apresentam maior adsorção de fosfatos que a caulinita (Novais \& Smyth, 1999). Dentre os óxidos, não há consenso na literatura sobre qual apresenta maior capacidade de adsorção de fosfatos. Fontes \& Weed (1996) encontraram maior adsorção de fosfatos em solos com predomínio de goethita, comparados a solos onde predominaram outros óxidos cristalinos. Entretanto, outro trabalho mostra que a hematita adsorveu mais fosfato (Gualberto et al., 1987). Portanto, quanto mais argiloso e mais oxídico for o solo, maior é a sua superfície de adsorção de fosfatos, particularmente nos Latossolos (Novais \& Smyth, 1999), e como conseqüência menores serão os valores de Prem na TFSA.

Se as frações silte e areia obtidas pelos métodos de rotina da análise textural fossem constituídas somente de minerais primários, principalmente partículas de quartzo, muscovita, biotita, feldspato, piroxênios e anfibólios, como afirmam Allen \& Hajek (1989), seria de esperar que contivessem baixa adsorção de fosfatos. Entretanto, os Latossolos apresentam microagregados do tamanho de silte, formados pela ação cimentante dos óxidos de $\mathrm{Fe}$ e $\mathrm{Al}$ mal cristalizados, aglutinando argilas. Esse fenômeno parece mais acentuado nos solos mais oxídicos (van Wambeke, 1962; Benemma et al., 1970; Moura Filho \& Buol, 1976; Resende, 1985; Kunze \& Dixon, 1986; Pede \& Langohr, 1986; van Wambeke, 1993; Galvão \& Schulze, 1996), o que leva, na análise textural, a superestimar a proporção de silte em detrimento da argila (Mitchell et al., 1964; Santana, 1973; Netto, 1996). Esses microagregados podem estar contribuindo para que a fração silte determinada no laboratório tenha uma relação mais 
acentuada com a retenção de água e de fosfatos dos Latossolos (van Wambeke, 1993; Netto, 1996). Pelo discutido anteriormente, seria razoável propor o Prem como uma estimativa da superfície de adsorção de fosfatos dos solos.

Diante do exposto, este trabalho objetivou avaliar o uso da determinação do Prem como medida da eficácia de pré-tratamentos na análise textural de Latossolos.

\section{MATERIAL E MÉTODOS}

Foram utilizadas amostras coletadas no horizonte A de sete Latossolos do Estado de Minas Gerais (Quadro 1), selecionados por apresentarem diferentes materiais de origem e teores elevados de óxidos de Fe (Donagemma et al., 2003).

Os tratamentos corresponderam a um fatorial $7 \times 2^{2}$, num delineamento em blocos casualizados, com quatro repetições. Os fatores em estudo foram as amostras de TFSA dos sete Latossolos indicados, que foram dispersas com $\mathrm{NaOH} 0,1 \mathrm{~mol} \mathrm{~L}^{-1}$, na ausência (ST) ou na presença $\left(2^{2}\right)$ de pré-tratamentos para remoção da MO (-MO) e dos óxidos de $\mathrm{Fe}$ e $\mathrm{Al}$ mal cristalizados (-Ox).

A remoção da MO foi realizada por oxidação com solução de $\mathrm{NaClO}$ 0,37 mol L ${ }^{-1}$ e relação solo:solução de 1:1,5 (Anderson, 1963); e a de óxidos de Fe mal cristalizados, com solução de $\mathrm{HCl} 10 \mathrm{mmol} \mathrm{L}^{-1} \mathrm{e}$ relação solo:solução de 1:5 (Barreto, 1986). Nas amostras em que foram removidos tanto a $\mathrm{MO}$ quanto os óxidos de $\mathrm{Fe}$ e $\mathrm{Al}$ mal cristalizados (-MO-Ox), utilizaram-se os mesmos reagentes, e a remoção da MO precedeu a dos óxidos.

Após a realização dos pré-tratamentos, as amostras foram secas em estufa a $45^{\circ} \mathrm{C}$. Retiraram-se subamostras para a determinação do teor de carbono de compostos orgânicos (Yeomans \& Bremner, 1988) e do teor dos óxidos de Fe mal cristalizados, após a extração com oxalato de amônio (McKeague \& Day, 1966). Essas determinações também foram realizadas nas amostras que não sofreram pré-tratamentos (Quadro 2).

Em seguida, procedeu-se à dispersão mecânica com agitação rápida, a 12.000 rpm, seguindo procedimento para separar as diferentes frações texturais, descrito por Donagemma et al. (2003). A fração silte das amostras dispersas com $\mathrm{NaOH}$ que não sofreram prétratamentos foi submetida à nova dispersão.

A determinação do Prem foi realizada em amostras da TFSA (Alvarez V. et al., 2001), das frações argila e silte da primeira dispersão, e da fração silte da segunda dispersão na solução de equilíbrio do solo com uma solução de $\mathrm{CaCl}_{2} 10 \mathrm{mmol} \mathrm{L}^{-1}$, contendo $60 \mathrm{mg} \mathrm{L}^{-1}$ de P. O tempo de agitação foi de $1 \mathrm{~h}$ e a relação solo:solução foi de 1:50 (Netto, 1996).

O Prem determinado após a primeira dispersão foi analisado estatisticamente, por meio da análise de variância, e os tratamentos dentro de cada solo foram comparados utilizando contrastes médios (Alvarez V. \& Alvarez, 2006), após verificação de homogeneidade de variância nos sete solos estudados. O Prem determinado após a segunda dispersão foi analisado, utilizando o teste de agrupamento de Scott-Knott.

\section{RESULTADOS E DISCUSSÃO}

Os valores de Prem das frações argila e silte, após a primeira dispersão, estão no quadro 3. É importante ressaltar que esses resultados não podem ser diretamente comparados com os valores comuns apresentados nas caracterizações de rotina. Nelas, utiliza-se a relação solo:solução de 1:10, enquanto para as frações estudadas a relação foi de 1:50. As frações argila e silte apresentam maior superfície de adsorção, em razão da sua composição mineralógica. A adsorção de $\mathrm{P}$ nessas frações é incrementada em relação à terra fina, e os valores do Prem na solução de equilíbrio

Quadro 1. Identificação e caracterização dos sete Latossolos

\begin{tabular}{|c|c|c|c|c|c|}
\hline \multirow{2}{*}{ Solo } & \multirow{2}{*}{ Classe de solo } & \multirow{2}{*}{ Silte $e^{(1)}$} & \multirow{2}{*}{ Argila ${ }^{(1)}$} & \multicolumn{2}{|c|}{$P$ remanescente } \\
\hline & & & & $1: 10^{(2)}$ & $1: 50^{(3)}$ \\
\hline & & \multicolumn{2}{|c|}{$-\mathrm{kg} \mathrm{kg}^{-1}$} & \multicolumn{2}{|c|}{$-\mathrm{mg} \mathrm{L}^{-1}$} \\
\hline $\mathrm{LVj}$ & Latossolo Vermelho perférrico & 0,36 & 0,15 & 2,4 & 38,2 \\
\hline LVdf1 & Latossolo Vermelho distroférrico & 0,24 & 0,56 & 2,8 & 32,1 \\
\hline LVdf2 & Latossolo Vermelho distroférrico & 0,17 & 0,63 & 4,1 & 36,9 \\
\hline LVdf3 & Latossolo Vermelho distroférrico & 0,19 & 0,54 & 9,4 & 50,0 \\
\hline LVAdf1 & Latossolo Vermelho-Amarelo distroférrico & 0,09 & 0,61 & 7,8 & 50,5 \\
\hline LVAdf2 & Latossolo Vermelho-Amarelo distroférrico & 0,15 & 0,73 & 3,9 & 36,8 \\
\hline $\mathrm{LVe}$ & Latossolo Vermelho eutrófico típico & 0,28 & 0,55 & 10,2 & 42,5 \\
\hline Média & & 0,21 & 0,54 & 5,8 & 41,0 \\
\hline
\end{tabular}

(1) Método da pipeta (Embrapa, 1997). ${ }^{(2)}$ Alvarez V. et al., 2001. ${ }^{(3)}$ Netto, 1996. 
Quadro 2. Teores de carbono de compostos orgânicos (CO) e ferro extraído com solução de oxalato de amônio (Fe ox) determinados em amostras não-tratadas (+MO+Ox) e nos materiais submetidos aos prétratamentos para remoção da matéria orgânica (-MO) e dos óxidos de ferro e alumínio mal cristalizados $(-\mathrm{Ox})$

\begin{tabular}{|c|c|c|c|c|c|c|c|c|}
\hline \multirow{2}{*}{ Solo } & \multicolumn{4}{|c|}{$\mathrm{CO}$} & \multicolumn{4}{|c|}{ Fe ox } \\
\hline & $+\mathrm{MO}+\mathrm{Ox}$ & $-\mathrm{MO}+\mathrm{Ox}$ & $+\mathrm{MO}-\mathrm{Ox}$ & $-M O-O x$ & $+\mathrm{MO}+\mathrm{Ox}$ & $-M O+O x$ & $+\mathrm{MO}-\mathrm{Ox}$ & $-M O-O x$ \\
\hline $\mathrm{LVj}$ & 4,12 & 1,80 & 4,02 & 1,79 & 1,11 & 1,09 & 0,91 & 0,92 \\
\hline LVdf1 & 3,28 & 1,36 & 3,18 & 1,34 & 0,42 & 0,42 & 0,39 & 0,36 \\
\hline $\mathrm{LVdf} 2$ & 3,24 & 1,71 & 3,20 & 1,72 & 0,45 & 0,44 & 0,42 & 0,42 \\
\hline LVdf3 & 2,44 & 1,34 & 2,42 & 1,31 & 0,63 & 0,62 & 0,61 & 0,60 \\
\hline LVAdf1 & 3,91 & 1,36 & 3,88 & 1,35 & 0,48 & 0,49 & 0,49 & 0,47 \\
\hline LVAdf2 & 3,18 & 1,05 & 3,14 & 1,04 & 0,37 & 0,36 & 0,32 & 0,29 \\
\hline $\mathrm{LVe}$ & 3,98 & 1,84 & 3,92 & 1,83 & 0,39 & 0,38 & 0,34 & 0,33 \\
\hline Média & 3,45 & 1,44 & 3,39 & 1,48 & 0,55 & 0,54 & 0,50 & 0,48 \\
\hline
\end{tabular}

podem se tornar extremamente baixos, se utilizada a relação habitual de 1:10 para as frações argila e silte.

Na comparação dos valores de Prem, a terra fina apresentou valores superiores aos das frações (Quadros 1 e 3). Isso era, logicamente, esperado na fração argila, mas não necessariamente no que diz respeito à fração silte. Esses valores indicam a presença dos microagregados de argila, conformando o pseudo-silte, com marcada adsorção de P (Donagemma et al., 2003).

Quando considerado o Prem na fração argila, em todos os casos, a remoção da MO incrementou a adsorção de $\mathrm{P}$, diminuindo o valor de Prem em relação aos observados na ausência de pré-tratamentos. Pelo contrário, a remoção dos óxidos de $\mathrm{Fe}$ e $\mathrm{Al}$ mal cristalizados provocou a redução da adsorção de $\mathrm{P}$, resultando em maiores valores de Prem (Quadro 3).

No caso da MO, sua remoção leva à maior exposição de superfícies de adsorção, pela diminuição das ligações dos complexos organominerais. De modo geral, quanto mais oxídico for o solo, a tendência é de incremento maior da adsorção, com conseqüente redução dos valores de Prem. A diminuição dos valores de Prem é maior quanto maior foi a remoção de MO (Quadro 2).

Com relação aos óxidos de Fe removidos, estes estariam depositados como um filme em volta dos minerais da fração argila de maior diâmetro (menor superfície específica). A elevada superfície específica dos óxidos de $\mathrm{Fe}$ mal cristalizados contribuiria sobremaneira para a adsorção de P. Sua remoção provocaria diminuição da adsorção e, conseqüentemente, aumento do valor de Prem (Quadro 3).

A aplicação conjunta dos pré-tratamentos gerou resultados diferenciados (Quadros 3 e 4). Para LVj, LVdf1, LVdf2 e LVe, a remoção da MO teve preponderância sobre a remoção dos óxidos de Fe, resultando em maior adsorção de P. Ao remover a
MO, expõem-se cargas positivas que vão adsorver o P. Para o LVdf3, o efeito da remoção dos óxidos foi mais acentuado, tendo sido observadas a diminuição da adsorção de $\mathrm{P}$ e a elevação dos valores de Prem. Este solo, depois do LVj, foi o que apresentou o maior teor de óxidos de $\mathrm{Fe}$ e Al mal cristalizados (Quadro 2). Já para o LVAdf1 e LVAdf2, os dois resultados opostos compensaram-se, sem produzir modificações no resultado final.

Esses resultados indicam que a composição e o teor de $\mathrm{MO}$ e de óxidos, bem como a associação dos dois, levaram a respostas diferenciadas dos solos à aplicação conjunta dos pré-tratamentos, o que indica que o fenômeno de adsorção não é controlado por uma única característica. Ressalta-se assim que, em geral, o Prem foi sensível em mostrar diferenças entre os prétratamentos aplicados, evidenciando alterações na proporção de superfície de adsorção. Tal fato indica que ele pode ser utilizado como determinação auxiliar em estudos de dispersão de solos.

Em relação à fração silte, o comportamento do Prem foi, em linhas gerais, semelhante ao das argilas (Quadros 3 e 4). Porém, as respostas foram menos marcantes e a adsorção de $\mathrm{P}$ diminuída em relação à evidenciada para as argilas, pois a fração silte desses solos tem menor superfície de adsorção com mistura de grãos simples (silte verdadeiro) e microagregados (pseudo-silte) (Donagemma et al., 2003). Os solos com menores valores de Prem apresentaram, em geral, maior proporção de pseudo-silte. Obviamente, o pseudo-silte leva a superestimar a adsorção de $\mathrm{P}$ pelo silte, que seria acentuadamente inferior se essa fração fosse constituída exclusivamente de grãos individuais, com menor superfície de adsorção.

Em todos os casos, a fração silte adsorveu menos $\mathrm{P}$ após a segunda dispersão (Quadros 3 e 5), tendo sido observados valores bem diferentes das relações do Prem na segunda dispersão em relação à primeira, 
isto é, 4,5 e 1,1 para o LVdf1 e o LVdf2, respectivamente. Porém, não houve correlação entre o incremento do Prem e a proporção do silte remanescente após a segunda dispersão $(r=$ $0,216 \mathrm{~ns})$, sendo nova evidência da resistência à dispersão diferenciada dos microagregados que permaneceram na fração silte.

O maior incremento do Prem para o LVdf1 está relacionado com a constituição predominantemente oxídica do pseudo-silte, evidenciada pelos valores de $\mathrm{Ki}$ e $\mathrm{Kr}$ relativamente baixos (Donagemma et al.,
2003), o que lhe confere maior estabilidade e contribui para o aumento de sua proporção na fração silte. Apesar de o LVdf1 recuperar menor proporção de argila do que o LVdf2, pelo fato de a composição de seus microagregados ser dominada por óxidos, ao proceder à segunda dispersão ocorreu, conseqüentemente, uma redução acentuada na adsorção, levando a maiores valores de Prem. Em contrapartida, para o LVdf2, obteve-se maior proporção de argila recuperada, em função da menor estabilidade dos agregados desse solo, que é conferida pela sua mineralogia predominantemente caulinítica, apesar de haver menor proporção desses agregados na fração silte (Donagemma et al., 2003).

Quadro 3. Fósforo remanescente (1:50) determinado nas frações argila e silte, após a primeira dispersão, em amostras não-tratadas (+MO+Ox) e nos materiais submetidos aos pré-tratamentos para remoção da matéria orgânica (-MO) e dos óxidos de ferro e alumínio mal cristalizados (-Ox)

\begin{tabular}{|c|c|c|c|c|c|c|c|c|}
\hline \multirow{2}{*}{ Solo } & \multicolumn{4}{|c|}{ Argila } & \multicolumn{4}{|c|}{ Silte } \\
\hline & $+\mathrm{MO}+\mathrm{Ox}$ & $-M O+O x$ & +MO-Ox & -MO-Ox & $+\mathrm{MO}+\mathrm{Ox}$ & $-M O+O x$ & +MO-Ox & -MO-Ox \\
\hline & \multicolumn{8}{|c|}{$\mathrm{mg} \mathrm{L}^{-1}$} \\
\hline $\mathrm{LVj}$ & 4,3 & 1,8 & 10,8 & 3,8 & 13,9 & 12,6 & 13,3 & 13,2 \\
\hline LVdf1 & 1,9 & 1,7 & 3,5 & 2,0 & 4,6 & 4,4 & 11,1 & 4,6 \\
\hline LVdf2 & 3,4 & 1,5 & 5,5 & 2,1 & 36,7 & 23,2 & 43,3 & 25,0 \\
\hline LVdf3 & 7,9 & 3,8 & 9,9 & 7,5 & 21,4 & 18,5 & 26,0 & 20,7 \\
\hline LVAdf1 & 6,5 & 5,7 & 7,0 & 6,4 & 35,4 & 33,9 & 41,3 & 49,2 \\
\hline LVAdf2 & 3,4 & 2,7 & 7,0 & 5,5 & 12,8 & 8,8 & 17,3 & 6,6 \\
\hline $\mathrm{LVe}$ & 8,7 & 1,7 & 11,2 & 2,8 & 35,6 & 29,4 & 52,1 & 55,2 \\
\hline Média & 5,2 & 2,7 & 7,8 & 4,3 & 22,9 & 18,7 & 29,2 & 24,9 \\
\hline
\end{tabular}

Quadro 4. Contrastes médios (Ci) do fósforo remanescente (1:50) determinado nas frações argila e silte, após a primeira dispersão, em amostras não-tratadas (+MO+Ox) e nos materiais submetidos aos prétratamentos para remoção da matéria orgânica (-MO) e dos óxidos de ferro e alumínio mal cristalizados $(-\mathbf{O x})$

\begin{tabular}{|c|c|c|c|c|c|c|}
\hline \multirow{2}{*}{ Tratamento } & \multicolumn{3}{|c|}{ Argila } & \multicolumn{3}{|c|}{ Silte } \\
\hline & $\mathbf{C}_{1}$ & $\mathbf{C}_{2}$ & $\mathrm{C}_{3}$ & $\mathbf{C}_{1}$ & $\mathbf{C}_{2}$ & $\mathrm{C}_{3}$ \\
\hline$+\mathrm{MO}+\mathrm{Ox}$ & -1 & -1 & 1 & -1 & -1 & 1 \\
\hline$-\mathrm{MO}+\mathrm{Ox}$ & 1 & -1 & -1 & 1 & -1 & -1 \\
\hline$+\mathrm{MO}-\mathrm{Ox}$ & -1 & 1 & -1 & -1 & 1 & -1 \\
\hline \multirow[t]{2}{*}{$-\mathrm{MO}-\mathrm{Ox}$} & 1 & 1 & 1 & 1 & 1 & 1 \\
\hline & \multicolumn{6}{|c|}{ Valores $\left(\mathrm{mg} \mathrm{L}^{-1}\right)$} \\
\hline $\mathrm{LVj}$ & $-4,8 * *$ & $4,3^{* *}$ & $-2,3^{* *}$ & $-0,7^{*}$ & 0,0 & 0,6 \\
\hline LVdf1 & $-0,9^{* *}$ & $1,0 * *$ & $-0,7 * *$ & $-3,4^{* *}$ & $3,4^{* *}$ & $-3,2^{* *}$ \\
\hline LVdf2 & $-2,7 * *$ & $1,4^{*}$ & $-0,8^{*}$ & $-15,9 * *$ & $4,2^{* *}$ & $-2,4^{* *}$ \\
\hline LVdf3 & $-3,3 * *$ & $2,9^{* *}$ & $0,9^{* *}$ & $-4,1^{* *}$ & $3,4^{* *}$ & $-1,2$ \\
\hline LVAdf1 & $-0,7$ & 0,6 & 0,1 & $3,2^{* *}$ & $10,6^{* *}$ & $4,7^{* *}$ \\
\hline LVAdf2 & $-1,1^{* *}$ & $3,2 * *$ & $-0,4^{*}$ & $-7,4^{* *}$ & $1,2^{* *}$ & $-3,4$ ** \\
\hline $\mathrm{LVe}$ & $-7,7$ ** & $1,8^{* *}$ & $-0,7^{*}$ & $-1,6 * *$ & $21,2^{* *}$ & 4,7 \\
\hline
\end{tabular}

$\mathrm{C}_{1}$ : remoção da $\mathrm{MO} ; \mathrm{C}_{2}$ : remoção de óxidos de ferro e alumínio mal cristalizados; $\mathrm{C}_{3}$ : interação remoção $\mathrm{MO}$ x remoção de óxidos de ferro e alumínio. * $\mathrm{e} * *$ : Significativo a 5 e $1 \%$, respectivamente, pelo teste $\mathrm{F}$. 
Quadro 5. Fósforo remanescente (1:50) determinado na fração silte após a segunda dispersão, e relação entre estes valores e os da primeira dispersão em amostras que não sofreram pré-tratamentos

\begin{tabular}{lccccccc}
\hline \multicolumn{1}{c}{ Determinação } & LVj & LVdf $\mathbf{1}$ & LVdf $\mathbf{2}$ & LVdf $\mathbf{3}$ & LVAdf $\mathbf{1}$ & LVAdf 2 & LVe \\
\hline Fósforo remanescente $\left(\mathrm{mg} \mathrm{L}^{-1}\right)^{(1)}$ & $33,1 \mathrm{c}$ & $20,9 \mathrm{~d}$ & $39,2 \mathrm{~b}$ & $33,7 \mathrm{c}$ & $39,7 \mathrm{~b}$ & $17,7 \mathrm{e}$ & $44,0 \mathrm{a}$ \\
Relação & 2,4 & 4,5 & 1,1 & 1,6 & 1,1 & 1,4 & 1,2 \\
\hline
\end{tabular}

(1) Letras diferentes indicam diferenças significativas para o fósforo remanescente pelo teste de agrupamento de Scott-Knott a $5 \%$.

Além disso, por serem colóides menos adsortivos, não foram verificados aumentos acentuados nos valores de Prem após a segunda dispersão, mesmo com a maior proporção de argila recuperada, o que evidencia que não só a proporção de argila na fração silte, mas, sobretudo, a constituição mineralógica da fração argila afeta a adsorção de fosfato, mostrando que o fenômeno é influenciado pela superfície do mineral e também pela carga.

\section{CONCLUSÕES}

O Prem varia com o pré-tratamento da análise textural empregado e com a mineralogia do solo. A maior remoção de argila pela segunda dispersão da fração silte nem sempre leva ao maior incremento no valor de Prem desta fração, evidenciando a resistência diferenciada dos microagregados de argila que permanecem na fração silte dos Latossolos estudados.

\section{LITERATURA CITADA}

ALLEN, B.L. \& HAJEK, B.F. Mineral occurrence in soil environments. In: DIXON, J.B. \& WEED, S.B., eds. Minerals in soil environments. Madison, Soil Science Society of America, 1989. p.200-209.

ALVAREZ V., V.H. Efecto de los factores cantidad, intensidad y capacidad amortiguadora de fosfato en la evolución del fósforo disponible, de suelos derivados de cenizas volcánicas de La Meseta Tarasca, Edo. Michoacán. Chapingo, Colegio de Potsgrado, 1982. 300p. (Tese de Doutorado)

ALVAREZ V., V.H. \& FONSECA, D.M. Definição de doses de fósforo para a determinação da capacidade máxima de adsorção de fosfato e para ensaios de casa de vegetação. R. Bras. Ci. Solo, 14:49-55, 1990.

ALVAREZ V., V.H.; DIAS, L.E.; RIBEIRO JÚNIOR, E.S.; SOUZA, R.B. \& FONSECA, C.A. Métodos de análises de enxofre em solos e plantas. Viçosa, MG, Universidade Federal de Viçosa, 2001. 131p.

ALVAREZ V., V.H. \& ALVAREZ, G.A.M. Comparação de médias por teste de hipóteses? Contrastes! B. Inf. SBCS, 31:24-34, 2006.
ANDERSON, J.U. An improved pretreatment for mineralogical analysis of samples containing organic matter. Clays Clay Miner., 10:380-388, 1963.

BACHE, B.W. \& WILLIAMS, E.G. A phosphate sorption index for soils. J. Soil. Sci., 22:289-301, 1971.

BAHIA FILHO, A.F.C. Índice de disponibilidade de fósforo em Latossolos do Planalto Central com diferentes características texturais e mineralógicas. Viçosa, MG, Universidade Federal de Viçosa, 1982. 179p. (Tese de Doutorado)

BENEMMA, J.; JONGERIUS, A. \& LEMOS, R.B. Micromorphology of some oxic and argillic horizons in south Brazil in relation to weathering sequences. Geoderma, 4:333-355, 1970.

BARRETO, W. Eletroquímica de solos tropicais de carga variável: capacidade da dupla camada elétrica. Itaguaí, Universidade Federal Rural do Rio de Janeiro, 1986. 273p. (Tese de Doutorado)

BHATTI, J.S.; COMEFORD, N.B. \& JOHTSON, C.T. Influence of oxalate and soil organic matter on sorption and desorption of phosphate onto a Spodic Horizon. Soil Sci. Soc. Am. J., 62:1089-1095, 1998.

DONAGEMMA, G.K.; RUIZ, H.A.; FONTES, M.P.F.; KER, J.C. \& SCHAEFER, C.E.G.R. Dispersão de Latossolos em resposta à utilização de pré-tratamentos na análise textural. R. Bras. Ci. Solo, 27:765-772, 2003.

FONTES, M.P.F. \& WEED, S.B. Phosphate adsorption by clays from Brazilian Oxisols: Relationships with specific surface area and mineralogy. Geoderma, 72:37-51, 1996.

FERNÁNDEZ R., I.E.J. Reversibilidade de fósforo não-lábil em diferentes solos, em condições naturais e quando submetidos à redução microbiológica ou química. Viçosa, MG, Universidade Federal de Viçosa, 1995. 94p (Tese de Doutorado)

GALVÃO, T.C.B. \& SCHULZE, D.G. Mineralogical properties of a collapsible lateritic soil from Minas Gerais, Brazil. Soil Sci. Soc. Am. J., 6:1969-1978, 1996.

GUALBERTO, V.; RESENDE, M. \& CURI, N. Química e mineralogia de Latossolos com altos teores de ferro, da Amazônia e do Planalto Central. R. Bras. Ci. Solo, 11:245252, 1987.

KER, J.C. Mineralogia, sorção e dessorção de fosfato, magnetização e elementos traços de Latossolos do Brasil. Viçosa, MG, Universidade Federal de Viçosa, 1995. 181p. (Tese de Doutorado) 
KUNZE, G.W. \& DIXON, J.B. Pretreatment for mineralogical analysis. In: KLUTE, A., ed. Methods of soil analysis. Part 1. Physical and mineralogical methods. 2 ed. Madison, American Society of Agronomy, Soil Science Society of America, 1986. p.91-99. (Agronomy Series, 5)

LOPEZ-HERNANDEZ, D.; SIEGERT, G. \& RODRIGUEZ, J.V. Competitive adsorption of phosphate with malate and oxalate by tropical soils. Soil Sci. Soc. Am. J, 50:14601462,1986

McKEAGUE, J.A. \& DAY, J.H. Dithionite and oxalateextractable $\mathrm{Fe}$ and $\mathrm{Al}$ as aids in differentiating various classes of soils. Can. J. Soil Sci., 46:13-22, 1966.

MITCHELL, B.O.; FARMER, V.C. \& McHARDY, W.J. Amorphous inorganic materials in soils. Adv. Agron., 16:327-383, 1964.

MOURA FILHO, W. \& BUOL, S.W. Studies of Latosol Roxo (Eutrustox) in Brazil: Micromorphology effect on ion release. Experientiae, 21:161-177, 1976.

MUNIZ, A.S. Disponibilidade de fósforo avaliada por extratores químicos e pelo crescimento da soja (Glycine $\max (\mathrm{L}$.) Merril) com diferentes fatores capacidade. Viçosa, MG, Universidade Federal de Viçosa, 1983. 79p. (Tese de Mestrado)

MUNIZ, A.S.; NOVAIS, R.F.; FREIRE, M.F.; NEVES, J.C.L. \& BARROS, N.F. Disponibilidade de fósforo e recomendação de adubação avaliadas por meio de extratores químicos e do crescimento de soja em amostras de solos com diferentes valores do fator capacidade. R. Ceres, 34:125$151,1987$.

NETTO, A.R. Influência da mineralogia nas propriedades físicoquímicas de solos brasileiros. Viçosa, MG, Universidade Federal de Viçosa, 1996. 144p. (Tese de Mestrado)

NOVAIS, R.F. Phosphorus supplying capacity of previously heavily fertilized soils. Raleigh, North Carolina State University, 1977. 153p. (Tese de Doutorado)

NOVAIS, R.F. \& SMYTH, T.J. Fósforo em solo e planta em condições tropicais. Viçosa, MG, Universidade Federal de Viçosa, 1999. 399p.
PEDE, K. \& LANGOHR, R. Microscopic study of pseudoparticles in dispersed soil samples. In: BULLOCK, P. \& MURPHY, C.D., eds. Soil micromorphology: Technique and applications. Berkhamsted, AB Academic Publishers, 1986. p.265-271.

RESENDE, J.M.A. Comparação de índices de sorção de fósforo no solo. Viçosa, MG, Universidade Federal de Viçosa, 1983. 79p. (Tese de Mestrado)

RESENDE, M. Aplicação de conhecimentos pedológicos à conservação de solos. Inf. Agropec., 128:3-18, 1985.

RUIZ, H.A. Incremento da exatidão da análise granulométrica do solo por meio da coleta da suspensão (silte+argila). R. Bras. Ci. Solo, 29:297-300, 2005.

SANTANA, D.P. Estudo de solos do Triângulo Mineiro e de Viçosa: I. Mineralogia, II. Adsorção de fosfatos. Viçosa, MG, Universidade Federal de Viçosa, 1973. 56p. (Tese de Mestrado)

SILVA, M.L.N.; CURI, N.; BLANCANUX, P.; LIMA, J.M. \& CARVALHO, A.M. Rotação adubo verde-milho e adsorção de fósforo em um Latossolo Vermelho-Escuro. Pesq. Agropec. Bras., 32:649-654, 1997.

VIOLANTE, A.; COLOMBO, C. \& BUODONNO, A. Competitive adsorption of phosphate and oxalate by aluminium oxides. Soil Sci. Soc. Am. J., 55:65-70, 1991.

van WAMBEKE, A.R. Criteria for classifying soils by age. J. Soil Sci., 1:124-132, 1962.

van WAMBEKE, A.R. Latosols. In: van WAMBEKE, A.R., ed. Soils of the tropics. Properties and appraisal. New York, McGraw Hill, 1993. p.140-160.

YEOMANS, J.C. \& BREMNER, J.M. A rapid and precise method for routine determination of carbon in soil. Comm. Soil Sci. Plant Anal., 19:1467-1476, 1988. 